\title{
Face symmetry assessment abilities: Clinical implications for diagnosing asymmetry
}

\author{
Tate H. Jackson ${ }^{a}$ [Proffit fellow], Stephen R. Mitroff ${ }^{b}$ [Associate professor], Kait Clark ${ }^{c}$ \\ [Doctoral candidate], William R. Proffit ${ }^{d}$ [Kenan professor], Jessica Y. Lee ${ }^{e}$ [Demeritt \\ professor], and Tung T. Nguyen ${ }^{f}$ [Clinical assistant professor] \\ aDepartment of Orthodontics, School of Dentistry, University of North Carolina, Chapel Hill, NC \\ ${ }^{b}$ Department of Psychology and Neurosciences, Center for Cognitive Neuroscience, Duke \\ University, Durham, NC; adjunct assistant professor, Department of Orthodontics, School of \\ Dentistry, University of North Carolina, Chapel Hill, NC \\ 'Department of Psychology and Neurosciences, Center for Cognitive Neuroscience, Duke \\ University, Durham, NC \\ dDepartment of Orthodontics, School of Dentistry, University of North Carolina, Chapel Hill, NC \\ eDepartment of Pediatric Dentistry, School of Dentistry, University of North Carolina, Chapel Hill, \\ NC \\ fDepartment of Orthodontics, School of Dentistry, University of North Carolina, Chapel Hill, NC
}

\begin{abstract}
Introduction-An accurate assessment of face symmetry is necessary for the development of a dentofacial diagnosis in orthodontics, and an understanding of individual differences in perception of face symmetry between patients and providers is needed to facilitate successful treatment.

Methods-Orthodontists, general dentists, and control participants completed a series of tasks to assess symmetry. Judgments were made on pairs of upright faces (similar to the longitudinal assessment of photographic patient records), inverted faces, and dot patterns. Participants completed questionnaires regarding clinical practice, education level, and self-confidence ratings for symmetry assessment abilities.
\end{abstract}

\begin{abstract}
Results-Orthodontists showed expertise compared with controls $(P<0.001)$, whereas dentists showed no advantage over controls. Orthodontists performed better than dentists, however, in only the most difficult face symmetry judgments $(P=0.006)$. For both orthodontists and dentists, accuracy increased significantly when assessing symmetry in upright vs inverted faces $(t=3.7, P$ $=0.001 ; t=2.7, P=0.02$, respectively).
\end{abstract}

Conclusions-Orthodontists showed expertise in assessing face symmetry compared with both laypersons and general dentists, and they were more accurate when judging upright than inverted

Copyright $\odot 2013$ by the American Association of Orthodontists.

Address correspondence to: Tate H.Jackson, CB \#7450, 262 Brauer Hall, University of North Carolina, Chapel Hill, NC 27599-7450; tatejackson@unc.edu.

All authors have completed and submitted the ICMJE Form for Disclosure of Potential Conflicts of Interest, and none were reported. 
faces. When using accurate longitudinal photographic records to assess changing face symmetry, orthodontists are likely to be incorrect in less than $15 \%$ of cases, suggesting that assistance from some additional technology is infrequently needed for diagnosis.

Symmetry is an important biologically based determinant of facial attractiveness, and assessment of symmetry in a patient's face is paramount to the development of a complete dentofacial diagnosis. ${ }^{1}$ In an era of modern orthodontics when the soft-tissue paradigm and patient perception often dictate the success of treatment outcomes, it is not acceptable for the orthodontist to simply identify problems and proceed with treatment. ${ }^{2}$ An understanding of individual differences in the perception of face symmetry across orthodontists, their patients, and other providers of dental care is needed to facilitate communication among these groups and to ensure optimal treatment results. To these ends, recent research has attempted to establish thresholds for the perception of a problem in face symmetry in various professional and nonprofessional groups.

Huisinga-Fischer et $\mathrm{al}^{3}$ asked participants to objectively compare asymmetry between subjects' faces. Their results suggest that orthodontists and surgeons might judge face symmetry more accurately than other groups but leave room for alternative explanations because of the nature of the stimuli and the tasks. Specifically, the face stimuli they used had pathologic deviations from normal symmetry and normal proportions, and the participants were asked to rate how the faces differed in terms of deformity from normal rather than in symmetry explicitly. Accordingly, the role of symmetry in the participants' judgments was unclear. Another study related to the perception of face symmetry suggested dental expertise, but the participants were asked to rate attractiveness rather than symmetry. ${ }^{4}$ Finally, a study using virtual 3-dimensional face stimuli suggested that whereas there are thresholds for the perception of an asymmetric nose or chin, orthodontists and oral surgeons showed no meaningful advantage in judging face symmetry compared with laypersons. ${ }^{5}$ None of these studies was designed to investigate expertise explicitly.

Of equal importance to the threshold for detection of a problem is an understanding of the orthodontist's perceptual ability in general and in relation to the patient. Are orthodontists experts at assessing face symmetry? To date, the data are equivocal at best because studies have not been adequately designed to answer that question. ${ }^{3-5}$ If an orthodontist is better at judging face symmetry than the patient, then he or she can confidently help the patient to decide whether treatment is warranted. If the orthodontist is not better at assessing face symmetry, then treatment outcomes as viewed by the patient might not meet the patient's goals. With the availability of 3-dimensional imaging of facial surfaces, which allows for the exact quantification of face symmetry, understanding perceptual differences might become increasingly relevant. ${ }^{6,7}$ If an orthodontist understands his or her abilities in relation to the patient, he or she might better be able to determine when such technology is needed to assist in diagnosis.

The aims of this study were to determine whether orthodontists possess expertise in assessing face symmetry and to explore the nature of this ability with the hope of informing clinical practice and patient communication. To accomplish these goals, we compared 
performance on symmetry judgment tasks across orthodontists, general dentists, and control participants with no training in face symmetry assessment.

\section{Material and Methods}

This study was considered exempt from institutional board review by the Office of Human Research Ethics at the University of North Carolina at Chapel Hill and approved as an addition to a separate ongoing study by the institutional review board of Duke University, Durham, NC. Orthodontists ( $n=31 ; 8$ women; mean age, 43.5 years; SD, 15.8 years) were recruited from the University of North Carolina's School of Dentistry, including residents in various stages of the 3-year program as well as full-time and part-time faculty members. The faculty participants reported an average of 27.4 years of clinical practice (SD, 12.5 years). General dentists were also recruited from the University of North Carolina's School of Dentistry and included residents and faculty members $(n=12 ; 3$ women; mean age, 53.1 years; SD, 13.2 years). Orthodontic residents, orthodontic faculty, and general dentists were all compensated $\$ 10$ per hour for their time.

Control participants with no symmetry training were recruited from 2 sources: nonprofessional laypersons from Duke University (nonprofessionals) and Transportation Security Administration (TSA) officers employed at Raleigh-Durham International Airport in North Carolina. The nonprofessionals represented a diverse cross-section of the Duke University community and included undergraduate, graduate, and postdoctoral students as well as employees and general affiliates of the university. This group of laypersons, with exclusion based only on vision screening or failure to give consent, had no known special visual skills. The nonprofessionals ( $\mathrm{n}=23$; 13 women; mean age, 20.9 years; SD, 4.5 years) were compensated with course credits or paid $\$ 10$ per hour for their participation. The TSA officers represented a population known to have enhanced visual cognition abilities unrelated to face symmetry assessment. ${ }^{8}$ The TSA officers $(n=10 ; 2$ women; mean age, 42.3 years; SD, 10.2 years) were not directly compensated because their data were collected during normal working hours as part of their employment. Participation in this study by the TSA officers was entirely confidential and voluntary. ${ }^{8}$ Two additional participants in the TSA group and 1 in the nonprofessional group had overall face accuracy scores that were 2 SD below the mean overall face accuracy score for all participants, and their data were excluded from all analyses. Exclusion of these data was carried out according to criteria established a priori to account for participants who might not make earnest judgments and instead just complete the tasks for the compensation provided. All participants had 20/20 vision or used corrective lenses at the time of data collection.

Data were acquired in 3 locations with identical protocols and environments: orthodontic resident, orthodontic faculty, and general dentist data were collected at the University of North Carolina's School of Dentistry; nonprofessional data were collected at Duke University in the Visual Cognition Laboratory; and TSA officer data were collected at Raleigh-Durham International Airport in a private testing room. The experiment was run in a dimly lit room; participants at the universities viewed the experiments on an Inspiron computer (Dell, Round Rock, Tex) with a 20-in cathode ray tube monitor, and participants at Raleigh-Durham airport viewed the experiments on Vostro 260 computers (Dell) and 23.6- 
in computer displays that were adjusted so that all participants were presented with stimuli of the same physical size. The participants were seated at a viewing distance of approximately $57 \mathrm{~cm}$ with no head restraint. Stimuli were presented and responses were recorded with MATLAB (MathWorks, Natick, Mass) using the Psychophysics Toolbox (version 3.0.8; http://psychtoolbox.org9-11). Questionnaire data were collected using the Qualtrics Research Suite (Qualtrics Labs, 2012; http://www.qualtrics.com/).

All participants completed 3 visual cognition tasks related to symmetry and presented in a blocked design; order was counterbalanced across all participants and tasks. Each task began with a series of practice trials, which were immediately followed by the experimental segment during which trial-by-trial accuracy and response time were recorded. At the start of each trial, a fixation cross was presented for $500 \mathrm{~ms}$, followed by the stimulus. Participants responded to each trial with 1 of 2 possible keys, and no feedback was provided.

Task 1 was symmetry assessment of upright faces. After 6 practice trials, the participants assessed symmetry in 96 trials of upright faces by making a 2-alternative forced-choice judgment between 2 versions of the same face presented side by side (Fig 1, A). Stimuli were presented on a black background, and the participant was instructed to press the " $\mathrm{z}$ " key if the face on the left appeared more symmetric or the " " key if the face on the right appeared more symmetric. Stimuli were presented until the participant responded. Trials were counterbalanced using a random number sequence assignment for each participant as to whether the right or left face was more symmetric in each trial. Stimuli consisted of black-and-white photographs of faces of 16 ( 8 female) white people captured using symmetric lighting and morphed to varying levels of overall face asymmetry while preserving averaged proportions. All points in each photograph were morphed simultaneously to change overall face symmetry, not individual features such as the nose or chin (see the study of Rhodes et al ${ }^{12}$ for details on stimuli generation). Veridical hairstyles (ie, the unaltered hairstyles) were maintained for all versions of each face by editing the original stimuli set from Rhodes et al with Photoshop Elements 10 (Adobe, San Jose, Calif) so that the hair could not be used as a cue to symmetry. Four versions of each face, varying in symmetry, were used: veridical (original) face, face with perfect symmetry, face with symmetry increased 50\% from veridical, and face with symmetry decreased 50\% from veridical (Fig 2). By pairing each face version with all iterations of that face, 6 possible pairings were created (veridical with perfect symmetry, veridical with high symmetry, veridical with low symmetry, high symmetry with perfect symmetry, high symmetry with low symmetry, and low symmetry with perfect symmetry). These stimuli were presented at random in terms of both the levels of symmetry being compared and the face that was used. Participants viewed all possible pairings (96 pairings in total) of each face during task 1.

Task 2 was symmetry assessment of inverted faces. It was identical to task 1, but all stimuli were presented upside down. The sequence of presentation was randomized separately from task 1.

Task 3 was symmetry detection in dot patterns. Participants judged whether a dot pattern presented as a centered image on a black background was perfectly symmetric about its vertical axis (Fig 1, B). Each dot image was displayed for $2000 \mathrm{~ms}$, after which the 
participants were asked to make a response using the "z" key to indicate that the dot pattern was symmetric or the "" key to indicate that the dot pattern was not symmetric. The 2000ms display time was used to maintain consistency with a previously used experimental protocol. ${ }^{13}$ Stimuli were 18-dot patterns based on the body patterns of animals with bilaterally symmetric bodies (see the study of Evans et al ${ }^{14}$ for details). After 4 practice trials, each dot pattern was presented in random order in both upright and inverted conditions, for a total of 36 trials.

Immediately after all the visual tasks, each participant completed a Web-based questionnaire that asked for demographic information, strategies used during the symmetry assessment, and subjective self-confidence ratings for the tasks completed with the Royal College of Physicians confidence rating scale (4, fully confident in most cases; 3 , confident in some cases; 2 , satisfactory but lacking confidence; 1 , not confident). ${ }^{15}$ The orthodontists and general dentists were asked whether the patient is most often upright or inverted when they assess face symmetry clinically and about their duration of training or clinical practice. The nonprofessionals and TSA officers were asked whether they had any training or experience in symmetry assessment.

\section{Results}

Descriptive statistics for accuracy, response time, and confidence ratings for each task are given in the Table. Normality of the data was confirmed using quantile-quantile plots, and homogeneity of variance between groups was confirmed using the Levene test. Accuracy and response times were compared among the groups using 1-way analysis of variance and the Tukey HSD test. Within-subject comparisons were made using paired $t$ tests or Wilcoxon signed rank tests (for confidence ratings); statistical significance was set at $P=$ 0.05 . Reliability measures for the face symmetry tasks have been reported previously, with Cronbach alpha coefficients greater than 0.92 in all cases. ${ }^{12}$

The orthodontists showed a statistically significant advantage in judging face symmetry overall $(F=6.6 ; P=0.001)$ and in both upright $(F=5.9 ; P=0.001)$ and inverted $(F=4.8 ; P$ $=0.004)$ face conditions compared with both the nonprofessionals and TSA officers (Tukey HSD, $P<0.05$ for all comparisons), but not compared with the general dentists. General dentists did not show a significant difference in accuracy overall for assessing symmetry in faces, whether upright or inverted, when compared with the nonprofessionals or the TSA officers. In the most difficult trials (when the differences in symmetry between faces were smallest: eg, perfect symmetry compared with high symmetry), orthodontists showed a significant advantage $(F=9.2 ; P<0.001)$ over general dentists $(P=0.01)$ as well as both nonprofessionals $(P<0.001)$ and TSA officers $(P=0.002)$.

There was a significant difference in response time overall $(F=7.2 ; P<0.001)$ and for both upright $(F=7.9 ; P<0.001)$ and inverted $(F=4.7 ; P=0.005)$ faces between orthodontists and general dentists compared with nonprofessionals (Tukey HSD, $P<0.02$ for all comparisons), but not between the orthodontists and general dentists compared with the TSA officers. Nonprofessionals took less time to judge symmetry than orthodontists, general dentists, or TSA officers. There were no other differences in response time among any 
groups. The total time to complete all 3 symmetry tasks averaged 21.6 minutes (SD, 12.9 minutes) across all participants.

There were no statistically significant differences in accuracy or response time for the dot stimuli among the groups for this control task.

Orthodontists showed within-subject differences in accuracy between each pair of tasks: upright vs inverted faces $(t=3.7 ; P=0.001)$, upright faces vs dot patterns $(t=10.7 ; P$ $<0.001)$, and inverted faces vs dot patterns $(t=10.6 ; P<0.001)$. General dentists also showed an advantage in accuracy for upright vs inverted faces $(t=2.7 ; P=0.02)$.

Orthodontists also demonstrated significant differences in confidence ratings for each pair of tasks: upright vs inverted faces $(\mathrm{z}=4.5 ; P<0.001)$, upright faces vs dot patterns $(\mathrm{z}=4.3 ; P$ $<0.001)$, and inverted faces vs dot patterns $(\mathrm{z}=2.5 ; P=0.01)$. No other group showed significant differences in confidence ratings for all pairs of tasks.

All but 4 participants in the orthodontist group $(n=31)$ and 2 in the general dentist group ( $n$ $=12$ ) reported clinically assessing symmetry with the patient upright. No nonprofessionals or TSA officers reported training or experience in judging face symmetry.

\section{Discussion}

Our results indicate that orthodontists have a clear advantage in assessing face symmetry compared with laypersons, and an advantage over general dentists in the most difficult cases. One might suggest that the orthodontists' enhanced performance is the result of motivation bias; they were simply trying harder because this was an evaluation of a skill that they knew they should possess. An appraisal of response time rules out that possibility (Fig 3 , B). Orthodontists took longer, on average, to respond when judging face symmetry than did the nonprofessionals. The TSA officers took just as long as the orthodontists, however, and they were significantly worse at assessing symmetry. Even though they took significantly longer to respond, the TSA group's accuracy matched that of the nonprofessionals. In short, increased response time-a logical indicator of motivation-does not equate to greater accuracy. Orthodontists truly appear to have an enhanced skill.

However, all participants, whether orthodontists, general dentists, or untrained controls, showed accuracy scores that indicated some aptitude in judging face symmetry. Even the lowest mean accuracy score of $78.4 \%$ (Table) represents a real increase above the $50 \%$ score one might expect from random chance alone because the face symmetry tasks all included only 2 possible responses. That all participants showed some skill might be due to the nature of faces and how humans tend to perceive them. Faces are ubiquitous visual stimuli, and behavioral and neuroimaging research has indicated that they are processed by special cognitive mechanisms in the brain that give an advantage in perceptual abilities when it comes to looking at and evaluating faces (see the study of Kanwisher and Yovel ${ }^{16}$ ). Face symmetry also is likely to be governed by special processes that provide a perceptual advantage compared with symmetry in nonface objects, such as dot patterns or teeth. ${ }^{17-19}$ 
One aspect of face symmetry processing that is of interest to orthodontists and general dentists is that the orientation of the face when symmetry is judged appears to have a significant effect. Both orthodontists and general dentists were significantly better at assessing symmetry in upright faces compared with inverted faces. This finding supports research reporting that when a face is inverted, it is not fully processed by the usual neural pathways of the brain that provide a perceptual advantage. ${ }^{17,19,20}$ It might be clinically meaningful that orthodontists and general dentists are better at judging symmetry when a face is upright. When administering dental care, the patient is often reclined, with the face inverted. Our data support the recommendation that the patient should be upright when face symmetry is assessed to achieve maximum accuracy. Interestingly, all but 4 orthodontists and 2 general dentists reported that their routine clinical practice included assessment of face symmetry with the patient upright rather than inverted.

It is important to remark that the clinical setting for which our data are most applicable involves the longitudinal comparison of patient photographs when the patient is being monitored for a progressive asymmetric deformity. Our experimental design, comparing 2 versions differing in symmetry of the same person's face, is most similar to the clinical activity of comparing accurate standardized photographic records taken over time for diagnostic purposes: to see whether the patient's face is becoming more asymmetric with time.

In an era when surface-scanning 3-dimensional technology allows computer-assisted assessment of face symmetry and changes in it over time, our data might also be an aid to understanding when technology such as this is needed. ${ }^{21,22}$ When a patient's records are compared over time, our data suggest that orthodontists will correctly identify symmetry in $87 \%$ of the cases, if the faces are viewed in an upright position using accurate photographs. So, perhaps less than $15 \%$ of these situations require the aid of additional tools, such as surface 3-dimensional superimpositions, to identify progressive aberrations in face symmetry.

Recent studies using 3-dimensional laser surface scanning estimate the overall asymmetry in normally developing child and adolescent faces to range on average from $8 \%$ to $68 \%$, with standard deviations as high as nearly $14 \% .^{6,7,23,24}$ Interestingly, our study used stimuli that varied the symmetry of the whole face from $50 \%$ less symmetric than the true face to perfect symmetry. Accordingly, the stimuli we used provide a reasonable representation of the range of asymmetry encountered in clinical practice, and our data give further insight to the nature of face symmetry judgment by both clinicians and laypersons.

The relative skill of all participants in our study has implications for patient communication. Providers should be aware that faces are unique visual stimuli, and that judging symmetry in faces is a perceptual process distinct from other tasks to evaluate dentofacial esthetics. ${ }^{25}$ Whereas orthodontists are experts at face symmetry assessment, patients are likely to possess some inherent skill as well. Similarly, orthodontists are better at judging face symmetry than general dentists, but only in difficult cases (eg, perfect vs high symmetry; Fig 2). Orthodontists can use this evidence as a framework for understanding the perceptual abilities of both patients and colleagues with whom they communicate. 
Orthodontists might also use our evidence as a basis for understanding their own abilities. Confidence ratings for symmetry tasks followed performance for this group (Fig 3, A and C); that is to say that orthodontists rated themselves most confident in the tasks for which they were most accurate and least confident in the tasks where they performed the worst. An ability to accurately evaluate one's own performance is an indicator of expertise ${ }^{26}$; consistent with this tenet, general dentists, non-professionals, and TSA officers failed to show the same pattern. Our evidence suggests that orthodontists' self-assessments of performance are more likely to be consistent with their actual accuracy than the selfassessments of untrained laypersons or general dentists making the same judgments. This finding might facilitate the reconciliation of differences in face symmetry perception between patient and provider.

\section{Conclusions}

Orthodontists demonstrate robust expertise in assessing face symmetry when compared with laypersons, and expertise in only the most difficult judgments compared with general dentists.

Both orthodontists and general dentists have a significant advantage when judging face symmetry with upright compared with inverted faces.

When face symmetry is assessed with accurate photographic records from different time points, analogous to monitoring a patient over time for a progressive asymmetric deformity, our data suggest that orthodontists will incorrectly identify symmetry in less than $15 \%$ of these situations.

\section{Acknowledgments}

We thank Gillian Rhodes and Christopher Evans for use of the visual stimuli files, and Caroline Albea, Sahsa Malinchoc, Shanley Lestini, Adam Biggs, and Elise Darling for assistance in data collection.

Supported in part by grant number T90DE-021986-01 from the National Institute of Dental and Craniofacial Research. The Transportation Security Administration data were collected through support from a subcontract with the Institute for Homeland Security Solutions, a research consortium sponsored by the Human Factors Division in the Department of Homeland Security. This material is based on work supported by the Department of Homeland Security under contract number HSHQDC-08-C-00100. Any opinions, findings, conclusions, or recommendations expressed in this material are those of the authors and do not necessarily reflect the official policy or position of the Department of Homeland Security or the United States government. This study is approved for public release.

\section{References}

1. Rhodes G. The evolutionary psychology of facial beauty. Annu Rev Psychol. 2006; 57:199-226. [PubMed: 16318594]

2. Proffit, WR.; Fields, HW.; Sarver, DM.; Ackerman, JL. Contemporary orthodontics. 5th. St Louis: Elsevier; 2012.

3. Huisinga-Fischer CE, Souren JP, v d Werken F, Prahl-Andersen B, van Ginkel F. Perception of symmetry in the face. J Craniofac Surg. 2004; 15:128-34. [PubMed: 14704579]

4. Naini FB, Donaldson AN, McDonald F, Cobourne MT. Assessing the influence of asymmetry affecting the mandible and chin point on perceived attractiveness in the orthognathic patient, clinician, and layperson. J Oral Maxillofac Surg. 2012; 70:192-206. [PubMed: 21571417]

5. Meyer-Marcotty P, Stellzig-Eisenhauer A, Bareis U, Hartmann J, Kochel J. Three-dimensional perception of facial asymmetry. Eur J Orthod. 2011; 33:647-53. [PubMed: 21355063] 
6. Djordjevic J, Toma AM, Zhurov AI, Richmond S. Three-dimensional quantification of facial symmetry in adolescents using laser surface scanning. Eur J Orthod. 2011 Jul 27. Epub ahead of print.

7. Primozic J, Perinetti G, Zhurov A, Richmond S, Ovsenik M. Assessment of facial asymmetry in growing subjects with a three-dimensional laser scanning system. Orthod Craniofac Res. 2012; 15:237-44. [PubMed: 23020694]

8. Biggs A, Cain M, Clark K, Darling E, Mitroff S. Assessing visual search performance differences between transportation security adiminstration officers and non-professional visual searchers. Visual Cognition. 2013 in press.

9. Brainard DH. The Psychophysics Toolbox. Spat Vis. 1997; 10:433-6. [PubMed: 9176952]

10. Pelli G. The VideoToolbox software for visual psychophysics: Transforming numbers into movies. Spat Vis. 1997; 10:437-42. [PubMed: 9176953]

11. Kleiner M, Brainard D, Pelli D. What's new in Psychtoolbox-3? Perception 36 ECVP Abstract Supplement. 2007

12. Rhodes G, Proffit F, Grady JM, Sumich A. Facial symmetry and the perception of beauty. Psychnomic Bulletin and Review. 1998; 5:659-69.

13. Oinonen KA, Mazmanian D. Facial symmetry detection ability changes across the menstrual cycle. Biol Psychol. 2007; 75:136-45. [PubMed: 17316956]

14. Evans CS, Wenderoth P, Cheng K. Detection of bilateral symmetry in complex biological images. Perception. 2000; 29:31-42. [PubMed: 10820589]

15. George JT, Warriner D, McGrane DJ, Rozario KS, Price HC, Wilmot EG, et al. Lack of confidence among trainee doctors in the management of diabetes: the trainees own perception of delivery of care (TOPDOC) diabetes study. QJM. 2011; 104:761-6. [PubMed: 21511736]

16. Kanwisher N, Yovel G. The fusiform face area: a cortical region specialized for the perception of faces. Philos Trans R Soc Lond B Biol Sci. 2006; 361(1476):2109-28. [PubMed: 17118927]

17. Rhodes G, Peters M, Lee K, Morrone MC, Burr D. Higher-level mechanisms detect facial symmetry. Proc Biol Sci. 2005; 272(1570):1379-84. [PubMed: 16006321]

18. Rhodes G, Peters M, Ewing LA. Specialised higher-level mechanisms for facial-symmetry perception: evidence from orientation-tuning functions. Perception. 2007; 36:1804-12. [PubMed: 18283930]

19. Chen CC, Kao KL, Tyler CW. Face configuration processing in the human brain: the role of symmetry. Cereb Cortex. 2007; 17:1423-32. [PubMed: 16923779]

20. Jones RM, Victor JD, Conte MM. Detecting symmetry and faces: separating the tasks and identifying their interactions. Atten Percept Psychophys. 2012; 74:988-1000. [PubMed: 22419373]

21. Kau CH, Zhurov A, Scheer R, Bouwman S, Richmond S. The feasibility of measuring threedimensional facial morphology in children. Orthod Craniofac Res. 2004; 7:198-204. [PubMed: 15562582]

22. Souccar NM, Kau CH. Methods of measuring the three-dimensional face. Semin Orthod. 2012; 18:187-92.

23. Djordjevic J, Pirttiniemi P, Harila V, Heikkinen T, Toma AM, Zhurov AI, et al. Three-dimensional longitudinal assessment of facial symmetry in adolescents. Eur J Orthod. 2011; 35:143-51. [PubMed: 21300725]

24. Primozic J, Perinetti G, Richmond S, Ovsenik M. Three-dimensional evaluation of facial asymmetry in association with unilateral functional crossbite in the primary, early, and late mixed dentition phases. Angle Orthod. 2012; 83:253-8. [PubMed: 22889202]

25. Masella RS, Meister M. The neuroanatomic basis of facial perception and variable facial discrimination ability: implications for orthodontics. Am J Orthod Dentofacial Orthop. 2007; 132:293-301. [PubMed: 17826596]

26. Kruger J, Dunning D. Unskilled and unaware of it: how difficulties in recognizing one's own incompetence lead to inflated self-assessments. J Pers Soc Psychol. 1999; 77:1121-34. [PubMed: 10626367] 

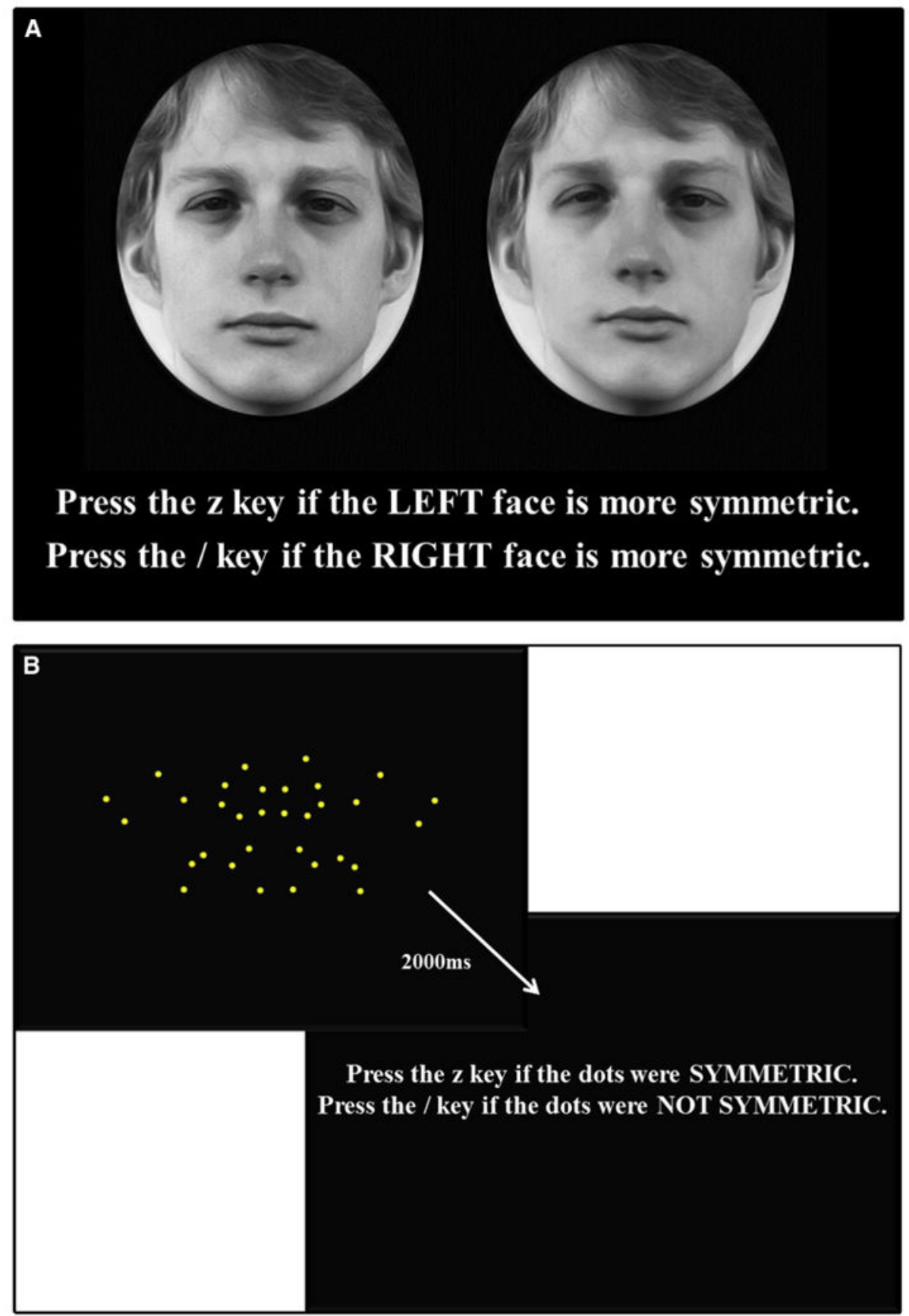

Fig 1.

Examples of stimuli and instructions presented to participants for $\mathbf{A}$, tasks 1 and 2 and $\mathbf{B}$, task 3. The face stimuli of tasks 1 and 2 were presented with no time constraints, whereas the dot patterns used in task 3 were presented for $2000 \mathrm{~ms}$ (followed by the instructions screen that remained until response). 


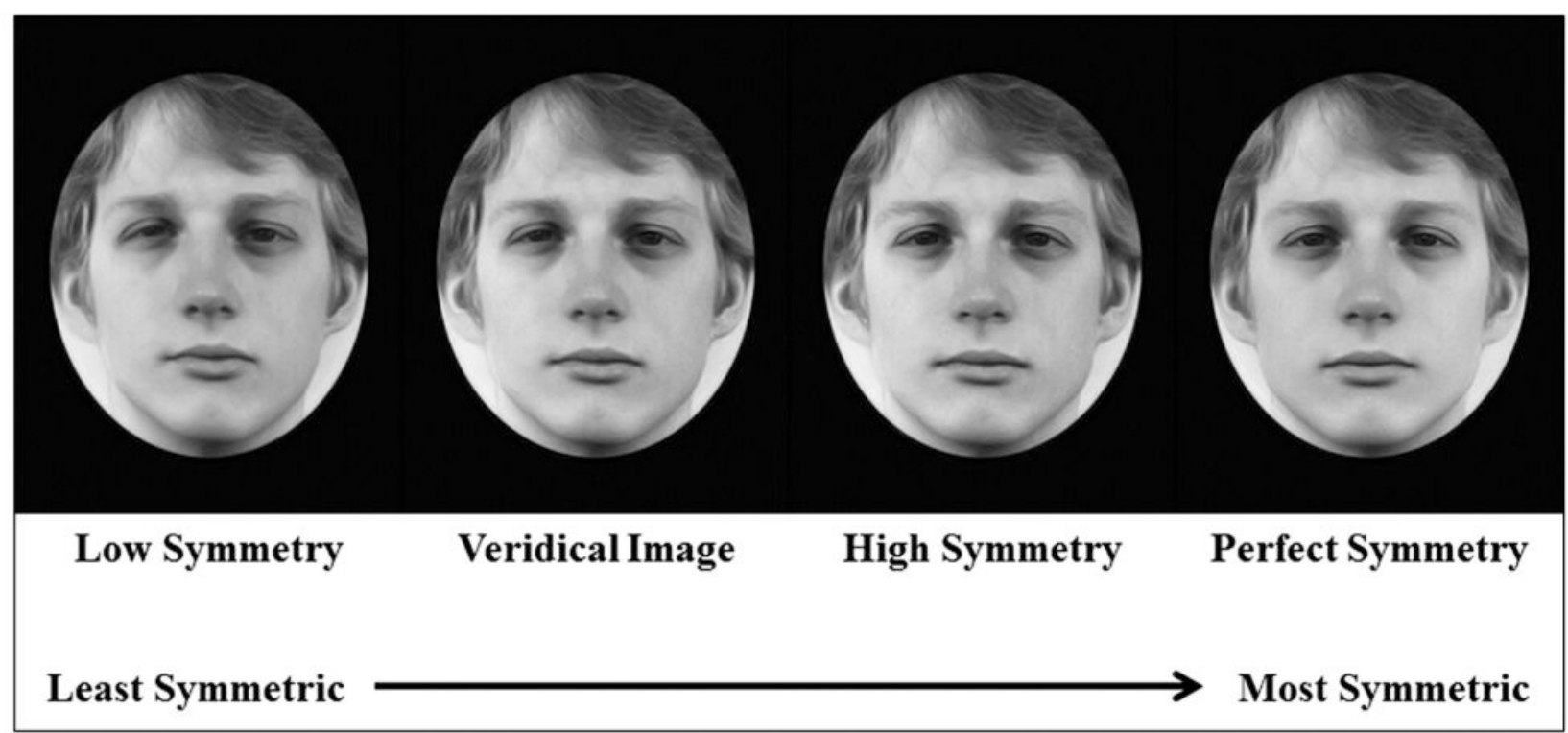

Fig 2.

Example of face stimuli showing 4 morphed versions of 1 person's face: the actual face (veridical), a version 50\% less symmetric (low symmetry), a version 50\% more symmetric (high symmetry), and a version with perfect symmetry. The hairstyle for each version of the face is the identical, veridical hairstyle. 

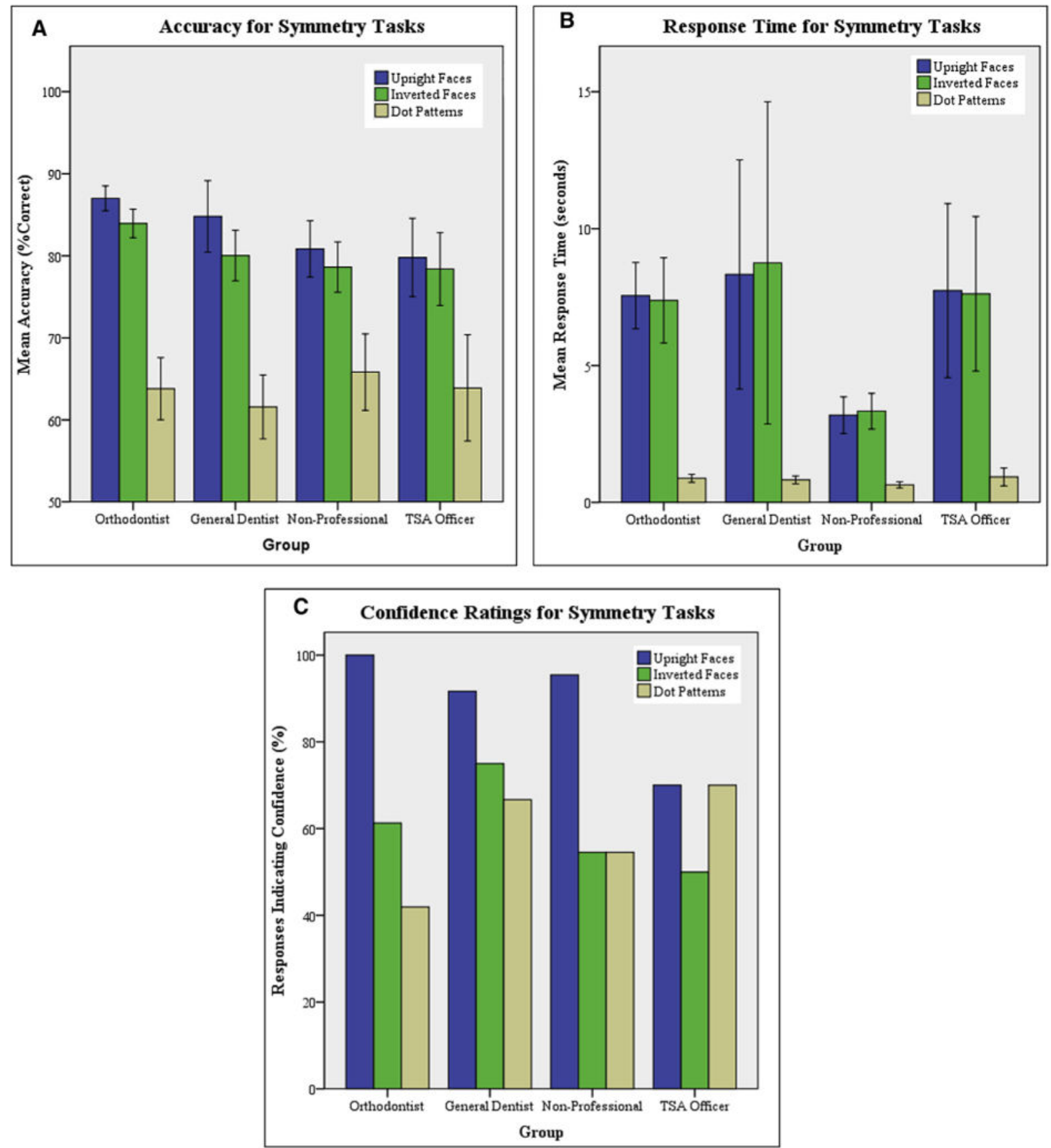

Fig 3.

A, Mean accuracy scores for each symmetry task (percent correct) by group. Error bars represent $95 \%$ confidence intervals. B, Mean response time for each symmetry task (in seconds) by group. Error bars represent $95 \%$ confidence intervals. Only dot pattern tasks had a restricted presentation time of $2000 \mathrm{~ms}$. C, Proportion of responses indicating confidence for each symmetry task by group. Royal College of Physicians confidence rating scale: responses indicating confidence: 4 , fully confident in most cases; and 3, confident in some 
cases; responses not indicating confidence: 2 , satisfactory but lacking confidence; and 1 , not confident. 
Table

Accuracy (percent correct), response time (seconds), and confidence scores for symmetry tasks

\begin{tabular}{|c|c|c|c|c|}
\hline & Orthodontists $(\mathrm{n}=\mathbf{3 1})$ & General dentists $(n=12)$ & Nonprofessionals $(\mathrm{n}=\mathbf{2 3})$ & TSA officers $(n=10)$ \\
\hline \multicolumn{5}{|c|}{ Accuracy, mean (SD) } \\
\hline All faces & $85.5(3.8)$ & $82.4(5.0)$ & $79.7(6.7)$ & $79.1(6.0)$ \\
\hline Upright faces & $87.0(4.1)$ & $84.8(6.8)$ & $80.8(7.9)$ & $79.8(6.7)$ \\
\hline Inverted faces & $84.0(4.7)$ & $80.0(4.8)$ & $78.6(7.1)$ & $78.4(6.2)$ \\
\hline Dots & $63.8(10.3)$ & $61.6(6.1)$ & $65.8(10.8)$ & $63.9(9.1)$ \\
\hline \multicolumn{5}{|c|}{ Response time, mean (SD) } \\
\hline All faces & $7.5(3.0)$ & $8.5(7.7)$ & $3.3(1.5)$ & $7.7(3.9)$ \\
\hline Upright faces & $7.6(3.3)$ & $8.3(6.6)$ & $3.2(1.6)$ & $7.7(4.4)$ \\
\hline Inverted faces & $7.4(4.2)$ & $8.8(9.3)$ & $3.3(1.5)$ & $7.6(4.0)$ \\
\hline Dots & $0.9(0.4)$ & $0.8(0.2)$ & $0.6(0.3)$ & $0.9(0.5)$ \\
\hline \multicolumn{5}{|c|}{ Confidence, percent of responses indicating confidence ${ }^{*}$} \\
\hline Upright faces & 100 & 91.7 & 91.3 & 70 \\
\hline Inverted faces & 61.3 & 75.0 & 52.1 & 50 \\
\hline Dots & 41.9 & 66.7 & 52.1 & 70 \\
\hline
\end{tabular}

* Royal College of Physicians confidence rating scale: 4, fully confident in most cases; 3 , confident in some cases; 2 , satisfactory but lacking confidence; 1 , not confident (responses of 3 or 4 indicate confidence). 